\title{
Computational models of human social behavior and neuroscience: An open educational course and Jupyter Book to advance computational training
}

\author{
Shawn A. Rhoads ${ }^{1}$ and Lin Gan ${ }^{1}$
}

1 Georgetown University

DOI: $10.21105 /$ jose. 00146

\section{Software}

- Review ¿

- Repository ca

- Archive c

Submitted: 28 September 2021 Published: 26 January 2022

\section{License}

Authors of papers retain copyright and release the work under a Creative Commons Attribution 4.0 International License (CC BY 4.0).

\section{Summary}

We present a semester-long educational course (see 14-week schedule) and Jupyter Book that provides introductory training in specifying, implementing, and interpreting computational models that characterize human social behavior and neuroscience. Through readings, discussions, and labs (e.g., Jupyter Notebook tutorials using Python), students will receive hands-on training in using mathematical models to test specific theories and hypotheses and explain unobservable aspects of complex social cognitive processes and behaviors. These aspects broadly include learning from and for others, learning about others, and social influences on decision-making and mental states.

Background in introductory psychological science and basic research methods/statistics is highly recommended prior to participating in this course. Programming experience is helpful, but not required or expected. Although the course content is tailored for undergraduate students, it was developed to be easily adapted for trainees at higher career stages.

\section{Statement of Need}

Developing formalized computational models is among the most promising approaches for advancing theories in psychological science and neuroscience (Guest \& Martin, 2021), including characterizing theories regarding the neural basis of social behavior (Cheong et al., 2017; Jolly \& Chang, 2017; Stanley \& Adolphs, 2013). This practice requires researchers to explicitly formalize scientific theories as quantifiable predictions that can be tested and falsified with empirical data. Computational models in research offer several advantages: they remove any ambiguity of verbal descriptions of theories, constrain the dimensionality of theories, shift focus away from null hypothesis significance testing in low-dimensional experimental designs (e.g., $2 \times 2$ factorial designs), and provide an open and reproducible approach (via programming code) for testing formal models of a theory (Guest \& Martin, 2021; Jolly \& Chang, 2017).

Despite the promise and increased use of computational models to characterize human social behavior and neuroscience (Zhang et al., 2020), few psychology programs actually offer quantitative training required for introductory level computational modeling and literacy (Guest \& Martin, 2021; Jolly \& Chang, 2017). Although summer schools (e.g., Neuromatch Academy (van Viegen et al., 2021), Methods in Neuroscience at Dartmouth (MIND), Neurohackademy, Cognitive Modeling Academy), self-guided online tutorials and papers (Lockwood \& Klein-Flügge, 2020; Wilson \& Collins, 2019; Zhang et

Rhoads et al., (2022). Computational models of human social behavior and neuroscience: An open educational course and Jupyter Book to 1 advance computational training. Journal of Open Source Education, 5(47), 146. https://doi.org/10.21105/jose.00146 
al., 2020), and community-oriented workshops (e.g., Brainhack Global (Craddock et al., 2016; Gau et al., 2021)) have filled this pedagogical gap in recent years, these programs may not reach audiences who are not familiar with computational modeling in research (e.g., undergraduates).

Therefore, we present course materials and accompanying Jupyter Book (Executable Books Community, 2020) to make training in computational modeling more accessible to both teachers and students in university settings and online.

\section{Learning Objectives}

Throughout this course, students are expected to meet the following goals:

- Understand the following: what are computational models, why use computational models, when to use computational models, and how models are fit to data

- Survey selected topics on social behavior through readings, discussions, and labs (e.g., Jupyter Notebook tutorials using Python)

- Learn how to write code for data science using the Python programming language and Jupyter Notebooks

- Fit mathematical models to data and evaluate their performance

- Interpret the results of social behavioral experiments

- Gain a richer understanding of how computational modeling advances psychological science and neuroscience

The course begins with readings and discussions on the utility of computational modeling in psychological science and neuroscience (see Statement of Need) and training in scientific Python (Module 01). Students will learn how to set up an Anaconda Environment and work in Google Colaboratory to run Python code in Executable Notebooks. They will then learn the basics of the Python programming language, including using numpy for scientific computing (Harris et al., 2020), pandas for data management (The Pandas Development Team, 2020), and matplotlib for data visualization (Hunter, 2007).

Then, students will learn the basics of modeling (Module 02). Using simulated data, they will learn how to fit linear and nonlinear models using least-squares minimization with a grid search, the scipy.optimize.minimize module (Virtanen et al., 2020), and the analytic solution for least-squares optimization. Using real data (O'Connell et al., 2021), they will learn how to estimate parameters (e.g., intercepts, slopes) from a linear model using both Ordinary Least Squares (OLS) and Maximum Likelihood Estimation (MLE). They will also learn how to assess model performance using $R^{2}$ and the Bayesian Information Criterion. They will compare their produced results to the actual results from the paper.

Next, students will learn the basics of reinforcement learning in non-social contexts (Module 03). They will learn how to run a "Two-Armed Bandit" experiment using PsychoPy (Peirce et al., 2019) and explore behavioral data from the task. Following exercises adapted from (Wilson \& Collins, 2019) and implemented in Python, students will simulate learning using three models: (1) random responding, (2) noisy win-staylose-shift, and (3) Rescorla-Wagner reinforcement learning. They will use MLE to fit reinforcement learning models to simulated data and recover model parameters (e.g., learning rate, temperature) (Palminteri et al., 2017).

During the second half of the course, students will apply what they have learned in the context of social experiences and interactions (Lockwood \& Klein-Flügge, 2020; Zhang et al., 2020) with an increased focus on reading empirical research papers with computational models, presenting findings and interpreting results in journal club settings, and discussing

Rhoads et al., (2022). Computational models of human social behavior and neuroscience: An open educational course and Jupyter Book to 2 advance computational training. Journal of Open Source Education, 5(47), 146. https://doi.org/10.21105/jose.00146 
with other students. Topics include learning about threats from others, learning how actions affect the outcomes of others, theory of mind, updating beliefs about others' states and traits, and social influences on decision-making. They will complete an adapted version of a social reinforcement learning task (Lockwood et al., 2016) in PsychoPy (Peirce et al., 2019) and develop models in Python to evaluate and compare the likelihood of each model given real data (Module 04). Finally, they will develop their own project and write a research proposal (formatted using guidelines for the National Science Foundation Graduate Research Fellowship Program Award application).

All course materials were designed to be modular. For example, if students are knowledgeable in Python basics and Jupyter Notebook (Module 01), then teachers could begin with model fitting (Module 02). If students are knowledgeable in model fitting with maximum likelihood estimation (Module 02), teachers could start with reinforcement learning (Module 03). Any tutorial can be adopted, transformed, or built upon for other educational purposes (e.g., courses, single class sessions, workshops) under a Creative Commons Attribution-ShareAlike 4.0 International License. Information on how teachers can use these materials can be found in the README documentation.

During the first iteration of the course in the spring 2021, students were $54.5 \%$ seniors (4th year), $36.4 \%$ juniors (3rd year), and 9.1\% sophomores (2nd year). Broadly, students reported that they enrolled in the course to learn how to use Python and computational modeling to accelerate psychological research, to learn how to work with data and research methodology beyond a traditional research methods \& statistics course, to learn skills relevant to a career in data science, and to gain a different perspective on topics from other classes. Most students reported spending 3-5 hours per week on readings and assignments (one student reported spending up to 10 hours and one student reported spending less than three hours). On a scale from 1 (strongly disagree) to 5 (strongly agree), students reported that the course was academically challenging and rigorous $(M=4.75, S D=0.43)$ and that the course materials $(M=4.00, S D=1.22)$, course-specific technologies $(M=4.00$, $S D=1.22)$, and assignments $(M=4.25, S D=0.83)$ helped them meet the stated learning objectives of the course.

\section{Tutorials \& Exercises}

\section{Module 01 - Scientific Python}

\begin{tabular}{lcc}
\hline & Run & View \\
\hline Tutorial: Jupyter Notebooks & Open in Colab & Open in Book \\
Tutorial: Python Basics & Open in Colab & Open in Book \\
Tutorial: Working with Data & Open in Colab & Open in Book \\
Exercise & Open in Colab & Open in Book \\
\hline
\end{tabular}

\section{Module 02 - Introduction to Modeling}

\begin{tabular}{lcc}
\hline & Run & View \\
\hline Tutorial: Linear Modeling & Open in Colab & Open in Book \\
Tutorial: Nonlinear Modeling & Open in Colab & Open in Book \\
Exercise & Open in Colab & Open in Book \\
\hline
\end{tabular}

Rhoads et al., (2022). Computational models of human social behavior and neuroscience: An open educational course and Jupyter Book to 3 advance computational training. Journal of Open Source Education, 5(47), 146. https://doi.org/10.21105/jose.00146 
Module 03 - Reinforcement Learning

\begin{tabular}{lcc}
\hline & Run & View \\
\hline Tutorial: Two-Armed Bandit & Open in Colab & Open in Book \\
Tutorial: Models of Learning & Open in Colab & Open in Book \\
Exercise & Open in Colab & Open in Book \\
\hline
\end{tabular}

\section{Module 04 - Social Learning}

\begin{tabular}{lcc}
\hline & Run & View \\
\hline Tutorial: Prosocial Learning & Open in Colab & Open in Book \\
Exercise & Open in Colab & Open in Book \\
\hline
\end{tabular}

Students have the option to interact with each tutorial notebook using one of two ways. The first option entails installing Anaconda 3 on their local computers with a custom environment made for this course and then opening each notebook using Jupyter Notebook. Teachers can walk students through the installation steps using the instructions provided on the Getting Started page. However, this can be rather difficult to implement with students' different machines, operating systems, background, etc. As a fail-safe, students also have the option to use Google Colaboratory, which runs the code on Google's cloud servers for free. There is a button at the top of each tutorial page that opens each Jupyter Book page in Google Colaboratory.

Once students are able to run notebooks (locally or on the cloud), they can interact with all of the code in the tutorials. Teachers should guide students through all of the "tutorials," which, for example, will allow them to demonstrate to students how tweaking certain variables in real-time would change the outputs. The "exercises" at the end of each module were created for the students to apply what they have learned. They can work on these by themselves or in groups. Links to example solutions for all exercises are provided on each page. In addition, teachers should familiarize themselves with the recommended readings associated with each module and use them to introduce students to each topic, generate discussions and ideas among students, and provide relevant background for each tutorial. For example, the Wilson \& Collins (2019) paper can be assigned for reading and discussion prior to beginning any notebook tutorials for Module 03.

For additional background on the content covered in each tutorial (technical or theoretical), teachers and students are encouraged to review the references and/or resources listed at the beginning of each tutorial or the non-exhaustive list of resources on the Resources page. Content-related questions from students, teachers, and contributors related to the course can also be submitted directly on the GitHub repository. However, we request that individuals make a good faith effort searching for their answer in the relevant readings or resources listed at the beginning of each tutorial before submitting questions.

\section{Future Directions}

This course will introduce trainees to using computational models for characterizing human social behavior and neuroscience. However, the covered topics are not exhaustive or comprehensive. We provide a growing list of literature that extends beyond the purview of the course. Furthermore, available tutorials only cover a selected range of topics. We welcome contributions from the community to extend the breadth and depth of tutorials.

Rhoads et al., (2022). Computational models of human social behavior and neuroscience: An open educational course and Jupyter Book to 4 advance computational training. Journal of Open Source Education, 5(47), 146. https://doi.org/10.21105/jose.00146 
These extensions may include improving or expanding existing content (e.g., stylistic improvements, alternative optimization algorithms), adding new tutorials on topics covered throughout the course, or adding new tutorials on related topics not covered throughout the course.

We appreciate any feedback on errors, omitted credit, or new ideas for this evolving project. These can be communicated on GitHub by either reporting an issue or requesting an enhancement. All contributions will be credited and should follow the community guidelines outlined on the Contribution page.

\section{Acknowledgments}

S. A. R. is grateful to Dr. Deborah Sterns, Joscelin Rocha-Hidalgo, and the Georgetown Center for New Designs in Learning \& Scholarship (CNDLS) for allowing him to use their teaching resources throughout the development of this syllabus. He is also grateful to Kelly C. Martin and Paige Amormino for reviewing initial drafts of this manuscript, Project Jupyter for making it possible to create and share these materials in a Jupyter Book (Executable Books Community, 2020), and all the students who participated during the first semester of this course (Spring 2021) for their insightful class contributions and feedback. The format of this course was largely inspired by the MIND Computational Summer School, Neuromatch Academy (van Viegen et al., 2021), Dr. Maximilian Risenhuber's Computational Neuroscience course, Dr. Robert C. Wilson's Modeling the Mind course, Dr. Luke J. Chang's DartBrains Jupyter Book (Chang et al., 2020), the research conducted in Dr. Abigail A. Marsh's Laboratory on Social and Affective Neuroscience, and the countless conversations with colleagues about the selected topics.

\section{Author Contributions}

S. A. R. designed and taught the course, generated the tutorials, assembled the Jupyter Book, reviewed the tutorials and contents of the Jupyter Book for bugs, typos, errors, stylistic enhancements, and clarity, revised contents of the Jupyter Book, and wrote the manuscript. S. A. R. learned to implement the relevant course methods through conducting research and participating in various summer courses (e.g., 2018 MIND Computational Summer School, 2018 Neuroscience School of Advanced Studies in Social Decision-Making, 2019 Computational Psychiatry Summer Course). He also received relevant pedagogical training as a Teaching Assistant (TA) for Neuromatch Academy, as a TA for Dr. Maximilian Risenhuber's Computational Neuroscience course, and as a participant in the Apprenticeship in Teaching Program at Georgetown University. The course materials were created to make the hands-on elements from these experiences as well as smaller, related workshops led by S. A. R. more accessible in university settings and online. L. G. participated in the course as a student in spring 2021, reviewed the tutorials and contents of the Jupyter Book for bugs, typos, errors, stylistic enhancements, and clarity, revised contents of the Jupyter Book, and wrote the manuscript.

\section{References}

Chang, L. J., Huckins, J., Cheong, J. H., Brietzke, S., Lindquist, M. A., \& Wager, T. D. (2020). DartBrains: An online open access resource for learning functional neuroimaging analysis methods in Python. Zenodo. https://doi.org/10.5281/zenodo. 3909717

Rhoads et al., (2022). Computational models of human social behavior and neuroscience: An open educational course and Jupyter Book to 5 advance computational training. Journal of Open Source Education, 5(47), 146. https://doi.org/10.21105/jose.00146 
Cheong, J. H., Jolly, E., Sul, S., \& Chang, L. J. (2017). Computational Models in Social Neuroscience (A. A. Moustafa, Ed.; pp. 229-244). John Wiley \& Sons. https://doi. org/10.1002/9781119159193.ch17

Craddock, R. C., Margulies, D. S., Bellec, P., Nichols, B. N., Alcauter, S., Barrios, F. A., Burnod, Y., Cannistraci, C. J., Cohen-Adad, J., Leener, B. D., Dery, S., Downar, J., Dunlop, K., Franco, A. R., Seligman Froehlich, C., Gerber, A. J., Ghosh, S. S., Grabowski, T. J., Hill, S., ... Xu, T. (2016). Brainhack: A collaborative workshop for the open neuroscience community. GigaScience, 5(16), 1-8. https://doi.org/10.1186/ s13742-016-0121-x

Executable Books Community. (2020). Jupyter Book. Zenodo. https://doi.org/10.5281/ zenodo. 2561065

Gau, R., Noble, S., Heuer, K., L., B. K., Bilgin, I. P., Yang, Y.-F., Huntenburg, J. M., Bayer, J., Bethlehem, R. A. I., Rhoads, S. A., Vogelbacher, C., Borghesani, V., Levitis, E., Wang, H.-T., Van Den Bossche, S., Kobeleva, X., Legarreta, J. H., Guay, S., Atay, S. M., ... The Brainhack Community. (2021). Brainhack: developing a culture of open, inclusive, community-driven neuroscience. Neuron, 109, 1-16. https: //doi.org/10.1016/j.neuron.2021.04.001

Guest, O., \& Martin, A. E. (2021). How Computational Modeling Can Force Theory Building in Psychological Science. Perspectives on Psychological Science, 16(4), 1-14. https://doi.org/10.1177/1745691620970585

Harris, C. R., Millman, K. J., Walt, S. J. van der, Gommers, R., Virtanen, P., Cournapeau, D., Wieser, E., Taylor, J., Berg, S., Smith, N. J., Kern, R., Picus, M., Hoyer, S., Kerkwijk, M. H. van, Brett, M., Haldane, A., Río, J. F. del, Wiebe, M., Peterson, P., ... Oliphant, T. E. (2020). Array programming with NumPy. Nature, 585(7825), 357-362. https://doi.org/10.1038/s41586-020-2649-2

Hunter, J. D. (2007). Matplotlib: A 2D graphics environment. Computing in Science $\mathscr{G}$ Engineering, 9(3), 90-95. https://doi.org/10.1109/MCSE.2007.55

Jolly, E., \& Chang, L. J. (2017). The flatland fallacy: Moving beyond low dimensional thinking. Topics in Cognitive Science, 11(2), 433-454. https://doi.org/10.1111/tops. 12404

Lockwood, P. L., Apps, M. A. J., Valton, V., Viding, E., \& Roiser, J. P. (2016). Neurocomputational mechanisms of prosocial learning and links to empathy. Proceedings of the National Academy of Sciences, 113(35), 9763-9768. https://doi.org/10.1073/ pnas.1603198113

Lockwood, P. L., \& Klein-Flügge, M. (2020). Computational modelling of social cognition and behaviour - a reinforcement learning primer. Social Cognitive and Affective Neuroscience, 16(8), 761-771. https://doi.org/10.1093/scan/nsaa040

O’Connell, K., Berluti, K., Rhoads, S., \& Marsh, A. (2021). Reduced social distancing during the COVID-19 pandemic is associated with antisocial behaviors in an online United States sample. PLoS ONE, 16(1), e0244974. https://doi.org/10.1371/journal. pone.0244974

Palminteri, S., Wyart, V., \& Koechlin, E. (2017). The Importance of Falsification in Computational Cognitive Modeling. Trends in Cognitive Sciences, 21(6), 425-433. https://doi.org/10.1016/j.tics.2017.03.011

Peirce, J., Gray, J. R., Simpson, S., MacAskill, M., Höchenberger, R., Sogo, H., Kastman, E., \& Lindeløv, J. K. (2019). PsychoPy2: Experiments in behavior made easy. Behavior Research Methods, 51(1), 195-203. https://doi.org/10.3758/s13428-018-01193-y

Stanley, D. A., \& Adolphs, R. (2013). Toward a neural basis for social behavior. Neuron, 80(3), 816-826. https://doi.org/10.1016/j.neuron.2013.10.038

Rhoads et al., (2022). Computational models of human social behavior and neuroscience: An open educational course and Jupyter Book to 6 advance computational training. Journal of Open Source Education, 5(47), 146. https://doi.org/10.21105/jose.00146 
The Pandas Development Team. (2020). Pandas. Zenodo. https://doi.org/10.5281/ zenodo.3509134

van Viegen, T., Akrami, A., Bonnen, K., DeWitt, E., Hyafil, A., Ledmyr, H., Lindsay, G. W., Mineault, P., Murray, J. D., Pitkow, X., Puce, A., Sedigh-Sarvestani, M., Stringer, C., Achakulvisut, T., Alikarami, E., Atay, M. S., Batty, E., Erlich, J. C., Galbraith, B. V., ... Peters, M. A. K. (2021). Neuromatch Academy: Teaching Computational Neuroscience with Global Accessibility. Trends in Cognitive Sciences, 25(7), 535-538. https://doi.org/10.1016/j.tics.2021.03.018

Virtanen, P., Gommers, R., Oliphant, T. E., Haberland, M., Reddy, T., Cournapeau, D., Burovski, E., Peterson, P., Weckesser, W., Bright, J., Walt, S. J. van der, Brett, M., Wilson, J., Millman, K. J., Mayorov, N., Nelson, A. R. J., Jones, E., Kern, R., Larson, E., ... Vázquez-Baeza, Y. (2020). SciPy 1.0: fundamental algorithms for scientific computing in Python. Nature Methods, 17(3), 261-272. https://doi.org/10. 1038/s41592-019-0686-2

Wilson, R. C., \& Collins, A. G. (2019). Ten simple rules for the computational modeling of behavioral data. eLife, 8, e49547. https://doi.org/10.7554/eLife.49547

Zhang, L., Lengersdorff, L., Mikus, N., Gläscher, J., \& Lamm, C. (2020). Using reinforcement learning models in social neuroscience: Frameworks, pitfalls, and suggestions. Social Cognitive and Affective Neuroscience, 15(6), 695-707. https://doi.org/10.1093/ scan/nsaa089 